\title{
Disease-Management-Programme in Deutschland: Erste Reaktionen der Diabetiker
}

\author{
M. Eller ${ }^{1}$ \\ W. Satzinger ${ }^{1}$ \\ R. Holle ${ }^{1}$ \\ C. Meisinger ${ }^{2}$ \\ B. Thorand ${ }^{3}$ \\ für die KORA-Studiengruppe
}

\author{
Disease Management Programmes in Germany: First Reactions by Patients \\ Suffering from Diabetes
}

\section{Zusammenfassung}

Die Versorgung von chronisch kranken Menschen, insbesondere auch von Diabetikern, im deutschen Gesundheitswesen gilt seit langem als defizient; ein erneuter Versuch, sie umfassend zu verbessern, sind die seit Sommer 2002 gesetzlich propagierten „strukturierten Behandlungsprogramme“ (DMP). Da die Teilnahme an ihnen freiwillig ist, hängt ihr möglicher Erfolg wesentlich von ihrer Bekanntheit und Akzeptanz bei den betroffenen Patientengruppen ab. Herauszufinden, mit welchen persönlichen Gegebenheiten die Resonanz von Diabetikern auf die DMP-Diskussion zusammenhängen könnte, war Ziel des hier vorgestellten Projekts. Dazu wurden 679 Diabetiker (Alter: 33 bis 88 Jahre) von November 2002 bis Dezember 2003 - d.h. in einem Zeitraum, noch bevor ihnen in Bayern ein konkretes DMP-Teilnahmeangebot gemacht worden war - im Rahmen einer Folgestudie der drei MONICA-Surveys S1 (1984/85), S2 (1989/90) und S3 (1994/95) in Augsburg (mehr als 13000 Probanden) schriftlich befragt. Die Stichprobe besteht überwiegend aus älteren Probanden (Mittelwert: 67,4 Jahre) mit längerer Diabetes-Dauer (Mittelwert: 9,8 Jahre). Nur 28\% der Befragten gaben an, bereits von Diabetes-DMP gehört zu haben, aber 54\% wären bereit, an einem derartigen Programm teilzunehmen, wenn ihnen Krankenkasse und Ärzteschaft dies vorschlagen würden. Als potenzielle Einflüsse auf diese Befragtenreaktionen wurden durch Regressionsanalysen u. a. ermittelt, dass das Interesse der Diabetiker an einer DMP-Teilnahme unter jenen von ihnen nachlässt, die durch kür-

\section{Abstract}

The performance of health delivery for chronic diseases in Germany has been considered deficient for a long time and in need of improvement. The launch of Disease Management Programmes (DMP) for type 2 diabetes in the summer of 2002 can be seen as an attempt to optimise health care for the chronically ill. The aim of our study was to find out at an early stage of DMP development which factors, personal or otherwise, would have an influence on whether diabetic patients know of, and are willing to participate in, such a programme. The data of 679 diabetic patients, collected between late 2002 and the end of 2003, presented here have been extracted from a written follow-up study to the three baseline surveys S1 (1984/85), S2 (1989/90) und S3 (1994/95) in Augsburg as part of the WHO-MONICA project with more than 13,000 participants. The study population is characterised by a rather high age (mean: 67.4 years), a long duration of the disease (mean: 9.8 years) as well as a high intensity of contacts with physicians. Only $28 \%$ of the diabetic patients had heard of the DMP, but a majority of the participants (54\%) stated their readiness to participate in DMP once offered. Higher age, low education as well as no participation in diabetes education have been identified in logistic regression models as impeding factors both for the knowing of and the participation in DMP. As a consequence, promotion of the DMP should be directed to those sections of the diabetic population outside the effective reach of the traditional diabetes care organisations.

Anmerkung

Die KORA-Studiengruppe besteht aus H.-E. Wichmann (Sprecher), H. Löwel, C. Meisinger, T. Illig, R. Holle, J. John und deren Mitarbeiter, die verantwortlich für das Design und die Durchführung der KORA-Studien sind.

Institutsangaben

${ }^{1}$ GSF- Forschungszentrum für Umwelt und Gesundheit, Institut für Gesundheitsökonomie und Management im Gesundheitswesen der GSF, Neuherberg

2 Zentralklinikum Augsburg, MONIKA/KORA Herzinfarktregister, Augsburg

${ }^{3}$ GSF- Forschungszentrum für Umwelt und Gesundheit, Institut für Epidemiologie, Neuherberg

Korrespondenzadresse

Martina Eller · GSF- Forschungszentrum für Umwelt und Gesundheit, Institut für Gesundheitsökonomie und Management im Gesundheitswesen der GSF · Ingolstädter Landstraße 1 · 85764 Neuherberg ·

E-mail: martina.eller@gmx.de

Bibliografie

Gesundheitswesen 2005; 67 Sonderheft 1: S144-S149 @ Georg Thieme Verlag KG Stuttgart · New York DOI 10.1055/s-2005-858255

ISSN 0949-7013 
zere Schulausbildung und höheres Alter, seltene Teilnahme an Diabetes-Schulungen und einen schlechteren Gesundheitszustand gekennzeichnet sind. Es wird daher darauf zu achten sein, dass speziell auch diesen Diabetikergruppen die neuen Programme nahe gebracht werden, damit sie nicht nur jenen chronisch Kranken zugute kommen, die mit ihrer Situation und auch dem bisherigen Versorgungsangebot ohnehin noch ziemlich gut zurechtkommen.

\section{Schlüisselwörter}

Diabetes mellitus · Disease-Management-Programme · Versorgungsforschung · Gesundheitssystem in Deutschland

\section{Hintergrund}

Disease-Management-Programme (DMP) sind der jüngste einer Reihe von Versuchen, die Organisation der Krankenversorgung im deutschen Gesundheitswesen so zu reformieren, dass sie das Prädikat, ein „System“ darzustellen, auch tatsächlich verdient. Vor allem die Koordinations- und Kooperationsmängel, die sowohl innerhalb der ambulanten (allgemein- oder fach-) ärztlichen Versorgung als auch zwischen ihr und der akut-stationären häufig auftreten und zu Diskontinuität und Inkonsistenz in der Patientenbehandlung führen, gelten seit langem als Ursachen schwerwiegender Qualitätsschwächen und Ineffizienzen des gesamten Versorgungsapparats.

Ab Mitte der 90er-Jahre nun gab es, in rascher Folge, mehrere gesetzliche Vorstöße und auch verbandliche Bemühungen, die beklagten ,Schnittstellenprobleme' besser zu bewältigen und Voraussetzungen für eine ,bruchlose' Krankenversorgung zu schaffen; Stichwörter: „Modellprojekte“, „Strukturverträge“ und „Integrierte Versorgung“ [1 - 4]. Das Gutachten des Sachverständigenrats von 2001 aber hielt diese Initiativen für noch unzureichend und monierte speziell, dass vor allem chronisch kranke Menschen wegen organisatorischer Mängel weiterhin einer gravierenden „Unterversorgung“ oder auch „Fehlversorgung“ ausgesetzt seien [5].

In Reaktion darauf sowie mit der Absicht, den 1993 eingeführten „Risikostrukturausgleich“ (RSA) zu reformieren [6], brachte im Sommer 2001 die Bundesregierung ein weiteres Reorganisationsmodell ins Spiel - „strukturierte Behandlungsprogramme“ für Krankheiten, für die Folgendes charakteristisch ist: hohe Prävalenz und Inzidenz des Krankheitsbildes, chronischer Verlauf mit definierten Krankheitsstadien, evidenzbasierte Versorgungsstandards, Messbarkeit klinischer Interventionsergebnisse, hohe Behandlungskosten, Bedarf an professions- und institutionsübergreifenden Versorgungskosten sowie Beeinflussbarkeit des Krankheitsverlaufs durch patienteneigene Verhaltenssteuerung. Für die Gestaltung der Programme wurde die Adaption einiger Struktur- und Prozesselemente der in amerikanischen ManagedCare-Organisationen entwickelten Disease-Management-Modelle empfohlen. Als Anreiz für die Krankenkassen, die Programme zu unterstützen, wird die Teilnahme ihrer Versicherten an ihnen positiv beim RSA berücksichtigt, womit chronisch Kranke für sie weniger ,unattraktiv’ würden und weiterer „Risikoselektion“" vorgebaut werde $[7,8]$.
Der Vorstoß der Bundesregierung erhielt eine gemischte Rezeption. Während die meisten Kassen (vor allem deren größte, die AOK) sich energisch für die Entwicklung der DMP einsetzten, reagierten Organisationen der Ärzteschaft (KBV, BÄK, HartmannBund) recht ablehnend auf sie. Hauptpunkte ihrer Kritik: Die Anbindung der DMP an den RSA verzerre Motive wie Ziele der Versorgung chronisch Kranker; die für die DMP obligaten Dokumentationen und deren Weitergabe an die Kassen verstießen gegen Datenschutz-Prinzipien (,gläserner Patient' und ,gläserner Arzt') und seien zudem eine unmäßige bürokratische Belastung der Arztpraxen; die in den DMP angelegten Versorgungsstandards schränkten die ärztliche Therapiefreiheit widersinnig ein, seien nichts anderes als „Verwaltungsdekrete einer kassengesteuerten Medizin“ (BKÄ-Präsident Hoppe) [9], zwängen zu einer schematisch angewandten ,Kochbuch-Medizin'.

Das letztgenannte Argumentationsbündel wurde besonders heftig vorgebracht, als im Frühjahr 2002 die ersten detaillierten Programmvorschläge für den Umgang mit jener Krankheit vorgestellt worden waren, die neben Brustkrebs von Anfang an fest zum Kreis der DMP-bedürftigen chronischen Erkrankungen gehörte, weil sie in epidemiologischer wie in gesundheitsökonomischer Hinsicht [10-14] die erwähnten Kriterien voll erfüllt: Diabetes mellitus. Gegen den Entwurf des Expertenausschusses machten sowohl der Diabetikerbund (DDB) als auch die Fachgesellschaft der Diabetologen (DDG) mobil: Seine Empfehlungen seien fachlich ein Fiasko - „Diese Form der Barfußmedizin wirft die (...) Fortschritte in der (...) Bekämpfung des Diabetes um ein Vierteljahrhundert zurück“" [15].

Dennoch setzte die Bundesregierung plangemäß zum 1.7.2002 die DMP-erweiterte Novelle zum RSA-Gesetz im Bundestag durch, und ab Mitte Dezember 2002 wurden die ersten DMPVerträge (in Sachsen-Anhalt und Baden-Württemberg) abgeschlossen; die meisten Regionen folgten im Frühjahr 2003 nach, allerdings waren daran selten alle Kassen und nicht einmal stets die jeweilige KV (sondern z. B. nur Gruppen niedergelassener Allgemeinärzte) beteiligt. Genau darauf - den neuen Vertrag mit der KVB und einheitlich für sämtliche Kassenarten abzuschließen - war man in Bayern bedacht; vor allem deshalb dauerte es bis zum November 2003, bis auch hier das Diabetes-DMP (als Nachfolger der seit 2000 gültigen „Diabetes-Vereinbarung“) vertraglich besiegelt war und mit der Werbung dafür bei der Ärzteund Patientenschaft begonnen werden konnte. 
Die grundsätzlichen Debatten über die Sinnhaftigkeit und Wirksamkeit der DMP sind mit diesen Vertragsabschlüssen freilich noch keineswegs beendet [16, 17]. Einer konkreten Auseinandersetzung darüber, ob denn die DMP überhaupt funktionieren und ob es jemandem nütze, wenn sie es tun, fehlt es ihrer kurzen Laufzeit wegen noch an empirischem Material. Ergebnisse amerikanischer Studien über die Effektivität von Diabetes-DMP [18-20] können nicht als übertragbar angesehen werden, und hierzulande gibt es dazu - außer eher methodisch-,theoretisch' angelegten Arbeiten [21, 22] - noch kaum einschlägige Studien.

Aus einer Umfrage bei ostdeutschen Ärzten wird von deren bisher sehr reservierter Resonanz auf das dort eingeführte Diabetes-DMP berichtet [23]. Freilich: Was die Ärzteschaft davon hält, ist selbstverständlich wichtig, aber doch nur die eine Seite der Erfolgsbedingungen dieser Programme. Deren Gelingen hängt mindestens ebenso von der Bereitschaft und den Erwartungen, später auch Erfahrungen, der Diabetiker selbst ab; schließlich ist ihnen die Teilnahme an einem DMP ja freigestellt, verlangt aber von ihnen dann, wenn einmal darin ,eingeschrieben', sehr rege und disziplinierte Mitarbeit. Deshalb sind Untersuchungen, die auch auf der Ebene der Patientenschaft - und zwar mit Primärdaten - die Akzeptanz der DMP analysieren, dringend gefragt [24].

\section{Thema und Methodik}

Thema der im Folgenden beschriebenen Studie ist die Wahrnehmung, die Diabetiker von, und die Einstellung, die sie zu den DMP haben, sowie ihre persönlichen Eigenschaften und Erfahrungen, mit denen ihre Haltung in beiderlei Hinsicht zusammenhängt. All das wird untersucht aus dem Blickwinkel eines Zeitpunkts (deshalb der ausführliche „Hintergrund“), als über diese Programme zwar öffentlich und kontrovers debattiert worden war, deren Inhalt und Form aber noch nicht detailliert gestaltet waren, also den betroffenen Kranken und ihren medizinischen Betreuern auch noch keine konkreten Offerten für ihre Teilnahme gemacht werden konnten. Das heißt, unsere Darstellung beschreibt gewissermaßen die patientenseitige Ausgangslage für die Einführung der Programme, fördert also auch Hinweise zutage, worauf zu achten sein wird, wenn die DMP vor allem jenen zugute kommen sollen, die es für die Bewältigung ihrer Krankheit besonders benötigen.

Die Daten stammen aus einer - im Rahmen der Kooperativen Gesundheitsforschung in der Region Augsburg (KORA) - von November 2002 bis Dezember 2003 durchgeführten bevölkerungsbezogenen, schriftlichen Befragung: einer Folgestudie zu drei MONICA Augsburg-Surveys aus den Jahren 1984/85 (S1), 1989/90 (S2) und 1994/95 (S3) mit insgesamt mehr als 13000 Personen, in der primär die Entwicklung des Gesundheitszustands dieser Menschen erfasst und darüber hinaus einige versorgungsrelevante Daten erhoben wurden.

Von den 9145 teilnehmenden Befragten (Alter: 33 bis 88 Jahre) wurden $756(=8,3 \%)$ als - größtenteils langjährige - Diabetiker identifiziert. Die speziell für diese Gruppe konzipierte Seite des Selbstausfüllfragebogens beantworteten $679(=90 \%)$ von ihnen. Sie enthielt - neben Fragen nach dem Grad der gesundheitlichen
Beeinträchtigung durch den Diabetes und der Intensität seiner aktuellen Behandlung - auch zwei DMP-spezifische: ob diese Diabetiker bereits die Bemühungen um die Entwicklung solcher Programme wahrgenommen hätten („Bekanntheit“) und - unabhängig davon! - ob sie sich prinzipiell für eine Teilnahme daran interessieren würden („Bereitschaft“).

Über zwei Drittel der diabetischen Probanden wurden bereits in den ersten beiden Monaten des 13-monatigen Erhebungszeitraums von der Befragung erreicht und sandten ihren Fragebogen ausgefüllt zurück. Die Antworten der restlichen, aus diversen Gründen schwerer zu kontaktierenden und für ihre Beteiligung zu motivierenden Probanden kamen erst im Laufe der folgenden elf Monate zurück, die Mehrheit von ihnen im ersten Halbjahr 2003.

Unsere Untersuchung versucht herauszufinden, welche Faktoren (soziodemographische Merkmale und Gesundheitszustand der Patienten, ihre Nutzung und Bewertung des bisherigen Versorgungsangebots u. a.) Einfluss auf die Haltung der Diabetiker zu solchen Programmen haben könnten. Bei der Analyse werden Chi-Quadrat-Tests sowie multivariate logistische Regressionsmodelle angewandt.

Diese Befragung selbst ist wieder ein Vorläufer zu der seit Februar 2004 laufenden KORA-F3-Studie (mehr hierzu in [25]). Darin werden Diabetiker in einem speziellen, noch ausführlicheren Selbstausfüllfragebogen um Auskunft über ihre Versorgungssituation, auch über ihre ersten Erfahrungen mit dem inzwischen eingeführten DMP, gebeten. Somit werden Mitte 2005 in einer kleinen Zeitreihe, reichend vom Spätherbst 2002 bis zum Frühjahr 2005, Informationen über die Diabetiker-Versorgung im Raum Augsburg aus Sicht der Betroffenen vorliegen.

\section{Ergebnisse}

In Tab. 1 wird die Verteilung einiger soziodemographischer und diabetesrelevanter Merkmale sowie Inanspruchnahme-Variablen in der untersuchten Diabetikerpopulation dargestellt. Angesichts des recht hohen Alters der Probanden und der Länge ihres Diabetiker-Status ist weder die Intensität ihrer Arztkontakte noch die Rate der bereits eingetretenen Folgeerkrankungen überraschend (vgl. Tab.1). Markante geschlechtsspezifische Unterschiede sind in den patientenbezogenen Merkmalen nicht festzustellen.

Die Antworten der Befragten zu ihrem Wissen von und ihrem Interesse an den angekündigten Diabetes-DMP sind folgende:

- $28 \%$ der befragten Diabetiker (29\% der Männer und 26\% der Frauen) geben an, von den Bemühungen um Diabetes-DMP schon gehört zu haben („Bekanntheit“); 7\% meinen, sie wissen nicht, ob das der Fall sei, der Rest (65\%; 64\% der Männer, $68 \%$ der Frauen) kennt die DMP offenbar nicht;

- über die Hälfte (54\%; 55\% der Männer und 53\% der Frauen) der Diabetiker bekundet ihr Interesse, an derartigen Programmen teilzunehmen („Bereitschaft“), darunter sind auch einige, die von den DMP-Planungen bisher noch nichts gehört haben. 18\% (17\% der Männer und 21\% der Frauen) erklären sich nicht 
bereit teilzunehmen und $28 \%$ ( $29 \%$ bzw. $26 \%$ ) zeigen sich noch unentschlossen („weiß nicht“);

- der Anteil der Personen, die von DMP gehört haben und an diesen auch teilnehmen möchten, ist größer (62\%) als der Anteil der Teilnahmebereiten ohne DMP-Kenntnis (52\%) $(p=0,02)$.

Abb.1 macht deutlich, dass Kenntnisstand und Teilnahmebereitschaft der Probanden je nach deren Lebensalter recht unterschiedlich sind: die älteste der drei Gruppen nimmt unter beiden Aspekten den letzten Rang ein, Wahrnehmung und Annahme der angekündigten Neuerungen liegen ihr am fernsten.

Tab. 2 beschreibt wahrscheinliche Zusammenhänge der Verbreitung von DMP-Bekanntheit und -Teilnahmebereitschaft der Befragten mit einigen ihrer Merkmale als Patienten und Personen. So ist beispielsweise zu sehen, dass Kenntnis von den Aktivitäten

Tab. 1 Merkmale der Diabetikerpopulation (gesamt und nach Geschlecht), Angaben in Prozent, Diabetiker der KORA-Follow-up-Befragung 2002/03 der MONICA-Surveys S1(1984/85), S2 (1989/90) und S3 (1994/95) in Augsburg

\begin{tabular}{|c|c|c|c|}
\hline Merkmale & $\begin{array}{l}\text { gesamt } \\
n=679\end{array}$ & $\begin{array}{l}\text { Männer } \\
n=397\end{array}$ & $\begin{array}{l}\text { Frauen } \\
n=282\end{array}$ \\
\hline Alter (unter 65 Jahre) & 41,2 & 42,8 & 39,0 \\
\hline $\begin{array}{l}\text { Schulbildungsabschluss } \\
\text { - kein Abschluss } \\
\text { - Hauptschule } \\
\text { - Realschule } \\
\text { - Abitur } \\
\text { - Hochschule/Universität }\end{array}$ & $\begin{array}{l}0,6 \\
74,7 \\
14,3 \\
3,1 \\
7,4\end{array}$ & $\begin{array}{l}- \\
72,3 \\
13,4 \\
3,0 \\
11,3\end{array}$ & $\begin{array}{l}1,4 \\
78,0 \\
15,6 \\
3,2 \\
1,8\end{array}$ \\
\hline $\begin{array}{l}\text { Diabetes-Dauer (bis } 5 \text { Jahre) } \\
\text { (min.: kürzer als } 1 \text { Jahr, max.: } 64 \text { Jahre) }\end{array}$ & 44,4 & 42,5 & 47,0 \\
\hline Teilnahme an Diabetiker-Schulung(en) & 50,5 & 48,4 & 53,5 \\
\hline $\begin{array}{l}\text { Zeitpunkt des letzten Arztbesuchs: } \\
\text { - innerhalb der letzten } 4 \text { Wochen } \\
\text { - innerhalb der letzten } 2 \text { bis } 12 \text { Monate } \\
\text { - vor mehr als einem Jahr } \\
\text { - weiß nicht }\end{array}$ & $\begin{array}{l}75,5 \\
23,2 \\
0,9 \\
0,5\end{array}$ & $\begin{array}{l}75,3 \\
23,2 \\
0,8 \\
0,8\end{array}$ & $\begin{array}{l}75,7 \\
23,2 \\
1,1 \\
-\end{array}$ \\
\hline $\begin{array}{l}\text { Behandlung }{ }^{1} \\
\text { - keine Behandlung } \\
\text { - nur mit Diät } \\
\text { - mit Tabletten } \\
\text { - mit Insulin } \\
\text { - mit Tabletten und Insulin }\end{array}$ & $\begin{array}{l}7,8 \\
14,3 \\
50,0 \\
17,3 \\
10,6\end{array}$ & $\begin{array}{l}8,3 \\
13,1 \\
51,0 \\
17,9 \\
9,6\end{array}$ & $\begin{array}{l}7,1 \\
16,0 \\
48,6 \\
16,3 \\
12,1\end{array}$ \\
\hline $\begin{array}{l}\text { Beurteilung der med. Versorgung: } \\
\text { - sehr gut } \\
\text { - gut } \\
\text { - teils-teils } \\
\text { - schlecht }\end{array}$ & $\begin{array}{l}24,5 \\
60,7 \\
13,4 \\
1,5\end{array}$ & $\begin{array}{l}25,3 \\
60,9 \\
12,4 \\
1,4\end{array}$ & $\begin{array}{l}23,4 \\
60,3 \\
14,8 \\
1,6\end{array}$ \\
\hline $\begin{array}{l}\text { Komplikationen (Nephro-, Retino- oder } \\
\text { Neuropathie) } \\
\text { - eine } \\
\text { - zwei } \\
\text { - drei }\end{array}$ & $\begin{array}{l}22,4 \\
6,2 \\
2,2\end{array}$ & $\begin{array}{l}22,3 \\
8,0 \\
3,0\end{array}$ & $\begin{array}{l}22,7 \\
3,7 \\
1,1\end{array}$ \\
\hline $\begin{array}{l}\text { selbsteingeschätzter Gesundheitszustand: } \\
\text { - sehr gut } \\
\text { - gut } \\
\text { - weniger gut } \\
\text { - schlecht }\end{array}$ & $\begin{array}{l}3,0 \\
55,6 \\
35,5 \\
6,0\end{array}$ & $\begin{array}{l}3,6 \\
57,0 \\
32,3 \\
7,1\end{array}$ & $\begin{array}{l}2,2 \\
53,5 \\
40,0 \\
4,4\end{array}$ \\
\hline
\end{tabular}

Abweichungen von $100 \%$ sind rundungsbedingt.

1 Bei dieser Variable waren ursprünglich Mehrfachantworten möglich. In der Tabelle schließen die Kategorien „mit Tabletten“, „mit Insulin“, „mit Insulin und Tabletten" ggf. auch diätetische Behandlung mit ein.

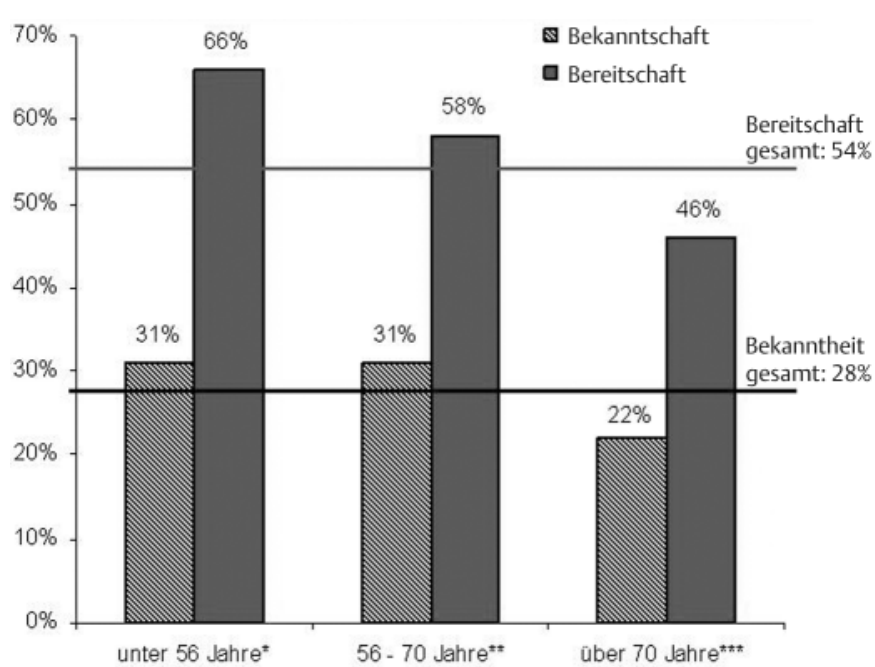

Abb. 1 Bekanntheit von und Bereitschaft zu Diabetes-DMP insgesamt und in Abhängigkeit vom Alter der Befragten, KORA-Follow-up-Befragung 2002/03 der MONICA-Surveys S1(1984/85), S2 (1989/90) und S3 (1994/95) in Augsburg.

$n$ (Gesamt) $=648$ (Bekanntheit) bzw. 642 (Bereitschaft), ${ }^{*} \mathrm{n}=80$ (Bekanntheit) bzw. 79 (Bereitschaft), ${ }^{* *} \mathrm{n}=313$ (Bekanntheit) bzw. 308 (Bereitschaft), ${ }^{* * *} \mathrm{n}=255$ (Bekanntheit und Bereitschaft).

$\mathrm{p} \leq 0,05$ (Bekanntheit) bzw. $\mathrm{p} \leq 0,001$ (Bereitschaft) (Chi-Quadrat-Test)

Tab. 2 Merkmale von Diabetikern und ihr Verhalten gegenüber DMP; Ergebnisse multivariater logistischer Regressionen, Diabetiker der KORA-Follow-up-Befragung 2002/03 der MONICA-Surveys S1(1984/85), S2 (1989/90) und S3 (1994/95) in Augsburg

\begin{tabular}{|c|c|c|}
\hline \multirow[b]{2}{*}{ Merkmale } & \multicolumn{2}{|c|}{$\begin{array}{c}\text { Verhalten } \\
\text { Odds Ratios (95\% KI) }\end{array}$} \\
\hline & $\begin{array}{l}\text { Bekanntheit der } \\
\text { DMP1: } \\
\text { ja vs. nein, } \\
n=582^{2}\end{array}$ & $\begin{array}{l}\text { Bereitschaft zu } \\
\text { DMP': } \\
\text { ja vs. nein, } \\
n=581^{2}\end{array}$ \\
\hline $\begin{array}{l}\text { Gesundheitszustand: } \\
\text { gut vs. schlecht }\end{array}$ & $\begin{array}{l}0,96 \\
(0,65-1,42)\end{array}$ & $\begin{array}{l}1,58 \\
(1,11-2,26)\end{array}$ \\
\hline $\begin{array}{l}\text { Dauer der Diabetes-Erkrankung: } \\
\text { bis } 5 \text { Jahre vs. } 6 \text { und mehr Jahre }\end{array}$ & $\begin{array}{l}1,1 \\
(0,74-1,64)\end{array}$ & $\begin{array}{l}1,37 \\
(0,96-1,97)\end{array}$ \\
\hline $\begin{array}{l}\text { Teilnahme an Diabetes-Schulungen: } \\
\text { ja vs. nein }\end{array}$ & $\begin{array}{l}1,62 \\
(1,09-2,41)\end{array}$ & $\begin{array}{l}1,41 \\
(0,98-2,02)\end{array}$ \\
\hline $\begin{array}{l}\text { Beurteilung der medizinischen } \\
\text { Versorgung: } \\
\text { gut vs. schlecht }\end{array}$ & $\begin{array}{l}2,31 \\
(1,23-4,34)\end{array}$ & $\begin{array}{l}0,47 \\
(0,28-0,78)\end{array}$ \\
\hline $\begin{array}{l}\text { Geschlecht: } \\
\text { männlich vs. weiblich }\end{array}$ & $\begin{array}{l}1,0 \\
(0,68-1,47)\end{array}$ & $\begin{array}{l}1,17 \\
(0,83-1,66)\end{array}$ \\
\hline $\begin{array}{l}\text { Alter: } \\
\text { bis } 65 \text { Jahre vs. } 66 \text { und mehr Jahre }\end{array}$ & $\begin{array}{l}1,5 \\
(1,02-2,21)\end{array}$ & $\begin{array}{l}1,38 \\
(0,98-1,97)\end{array}$ \\
\hline $\begin{array}{l}\text { schulische Ausbildung: } \\
\text { höherer Abschluss vs. Hauptschule } \\
\text { und kein Abschluss }\end{array}$ & $\begin{array}{l}2,15 \\
(1,43-3,23)\end{array}$ & $\begin{array}{l}2,23 \\
(1,49-3,34)\end{array}$ \\
\hline
\end{tabular}

Lesebeispiel: Befragten mit einem höheren Schulabschluss sind die DMP eher bekannt als solchen mit einem niedrigeren (Odds ratio $=2,15$ ). Die Ergebnisse in den schattierten Zellen sind statistisch signifikant.

${ }^{1}$ Die Antwortkategorien „weiß nicht“ und "nein“ wurden in den logistischen Regressionsmodellen zu einer Kategorie zusammengefasst.

297 bzw. 98 Probanden wurden aufgrund von fehlenden Werten in einer oder mehrerer der relevanten Variablen aus dieser Analyse ausgeschlossen. 
für Diabetes-DMP (erste Spalte) nicht nur bei jüngeren Diabetikern eher zu erwarten ist als bei älteren (OR: 1,5), sondern auch bei denen mit höherem Schulabschluss $(2,15)$ oder Teilnahme an Diabetes-Schulungen $(1,62)$ als bei der jeweiligen Vergleichsgruppe. Besonders hoch ist die Wahrscheinlichkeit, von den DMP-Planungen gehört zu haben, unter den Diabetikern, die über ihre medizinische Versorgung ein vergleichsweise positives Urteil haben (Odds ratio: 2,31).

Eine höhere Teilnahmebereitschaft an DMP (zweite Spalte) wird von Patienten geäußert, die jünger, gebildeter, subjektiv gesünder, weniger lange an Diabetes erkrankt sind und bereits an Schulungen teilgenommen haben. Aber auch die Unzufriedenheit mit der bisherigen medizinischen Versorgung ist ein signifikanter Faktor für die Akzeptanz von DMP.

Im Übrigen spielt das Geschlecht der Befragten bei der Reaktion auf beide Fragen offenbar keine, die Dauer ihrer Diabeteserkrankung nur eine geringe Rolle.

Die in Tab. 3 wiedergegebenen Berechnungsergebnisse beleuchten diese Zusammenhänge zwischen Patientenmerkmalen und DMPbezogenem Verhalten aus einer noch spezielleren Perspektive: nämlich welche von den schon dargestellten Patientenmerkmalen am engsten mit der DMP-Teilnahmebereitschaft zusammenhängen. Hierzu sind die befragten Diabetiker in zwei Gruppen zusam-

Tab. 3 DMP-Bereitschaft (abhängige Variable) in Abhängigkeit von patientenbezogenen Merkmalen bei Befragten mit und ohne DMPKenntnis; Ergebnisse multivariater logistischer Regressionen, KORAFollow-up-Befragung 2002/03 der MONICA-Surveys S1 (1984/85), S2 (1989/90) und S3 (1994/95) in Augsburg

\begin{tabular}{|c|c|c|}
\hline \multirow[b]{2}{*}{ Merkmale } & \multicolumn{2}{|c|}{$\begin{array}{l}\left.\text { Teilnahmebereitschaft (ja vs. nein }{ }^{1}\right) \\
\text { Odds Ratios ( } 95 \% \text { KI) }\end{array}$} \\
\hline & $\begin{array}{l}\text { Bekanntheit: ja } \\
n=157^{2}\end{array}$ & $\begin{array}{l}\text { Bekanntheit: } \\
\text { nein }^{1}, n=414^{2}\end{array}$ \\
\hline $\begin{array}{l}\text { Gesundheitszustand: } \\
\text { gut vs. schlecht }\end{array}$ & $\begin{array}{l}1,27 \\
(0,63-2,59)\end{array}$ & $\begin{array}{l}1,79 \\
(1,16-2,75)\end{array}$ \\
\hline $\begin{array}{l}\text { Dauer der Diabetes-Erkrankung: } \\
\text { bis } 5 \text { Jahre vs. } 6 \text { und mehr Jahre }\end{array}$ & $\begin{array}{l}0,98 \\
(0,47-2,08)\end{array}$ & $\begin{array}{l}1,56 \\
(1,02-2,4)\end{array}$ \\
\hline $\begin{array}{l}\text { Teilnahme an Diabetes-Schulungen: } \\
\text { ja vs. nein }\end{array}$ & $\begin{array}{l}0,74 \\
(0,35-1,58)\end{array}$ & $\begin{array}{l}1,72 \\
(1,12-2,63)\end{array}$ \\
\hline $\begin{array}{l}\text { Beurteilung der medizinischen } \\
\text { Versorgung: gut vs. schlecht }\end{array}$ & $\begin{array}{l}0,45 \\
(0,11-1,83)\end{array}$ & $\begin{array}{l}0,41 \\
(0,23-0,72)\end{array}$ \\
\hline $\begin{array}{l}\text { Geschlecht: } \\
\text { männlich vs. weiblich }\end{array}$ & $\begin{array}{l}1,07 \\
(0,53-2,18)\end{array}$ & $\begin{array}{l}1,21 \\
(0,81-1,83)\end{array}$ \\
\hline $\begin{array}{l}\text { Alter: } \\
\text { bis } 65 \text { Jahre vs. } 66 \text { und mehr Jahre }\end{array}$ & $\begin{array}{l}1,98 \\
(0,98-4,0)\end{array}$ & $\begin{array}{l}1,18 \\
(0,77-1,79)\end{array}$ \\
\hline $\begin{array}{l}\text { schulische Ausbildung: } \\
\text { höherer Abschluss vs. Hauptschule } \\
\text { und kein Abschluss }\end{array}$ & $\begin{array}{l}2,2 \\
(1,04-4,66)\end{array}$ & $\begin{array}{l}2,09 \\
(1,26-3,47)\end{array}$ \\
\hline
\end{tabular}

Lesebeispiel: Unter den 157 Personen mit DMP-Kenntnis sind an einer DMP-Teilnahme eher jene mit einem höheren Schulabschluss interessiert als solche mit einem niedrigeren (Odds ratio =2,2). Die Ergebnisse in den schattierten Zellen sind statistisch signifikant.

${ }^{1}$ Die Antwortkategorien „weiß nicht“ und „nein“ wurden in den logistischen Regressionsmodellen zu einer Kategorie zusammengefasst.

222 bzw. 55 Probanden wurden aufgrund von fehlenden Werten in einer oder mehrerer der relevanten Variablen aus dieser Analyse ausgeschlossen. mengefasst, je nachdem, ob sie von den in Vorbereitung befindlichen DMP wissen oder nicht. In der relativ großen Untergruppe (72\%) derer, denen DMP nicht bekannt sind, bilden sich die in Tab. 2 dargestellten Zusammenhänge ähnlich oder sogar noch deutlicher ab. Bei der kleineren Gruppe mit Vorwissen über DMP sind außer bei den Variablen Bildung, Alter und Beurteilung der Versorgung die Zusammenhänge weniger stark.

\section{Diskussion}

Der Bekanntheitsgrad von Initiativen für Diabetes-spezifische „strukturierte Behandlungsprogramme“ war unter den Diabetikern im Raum Augsburg Ende 2002/Anfang 2003, also einige Monate vor dem konkreten Programm-Angebot in Bayern, erwartungsgemäß gering (Abb.1); Befragte, die davon wussten, hatten wohl die lebhafte bundesweite Diskussion über derartige Pläne verfolgt.

Noch am ehesten informiert zeigten sich die Jüngeren und länger Ausgebildeten unter den Diabetikern und die, die bereits an Diabetes-Schulungen teilgenommen hatten und mit ihrer bisherigen Versorgung recht zufrieden waren. Wiederholt sich hier also das aus vielen Präventions- und Gesundheitsförderungsprogrammen vertraute Muster, dass sie vor allem von Personen wahrgenommen werden, die ohnehin schon zu den gesundheitlich agileren Bevölkerungsschichten zählen und die bereits vorhandene Versorgung gut nutzen? Mithin ein weiterer Fall von ,Predigten an die bereits Bekehrten'?

Auch die grundsätzliche Bereitschaft, an einem solchen Diabetiker-Programm teilzunehmen, wenn es ihnen denn von den Krankenkassen und Kassenärzten offeriert würde, war in der so charakterisierten Personengruppe deutlich größer - allerdings mit einer markanten Ausnahme: Diejenigen diabetischen Patienten, die mit der medizinischen Versorgung bis dato eher unzufrieden waren, äußerten eine wesentlich stärkere Neigung, sich auf das neue Behandlungsmodell einzulassen als ihre Leidensgenossen, die sich schon gegenwärtig gut versorgt fühlten.

Das leuchtet natürlich ein und ist zudem gesundheitspolitisch sinnvoll: den sich nach eigenem Urteil unzureichend behandelten Kranken eine Alternative bieten! Den Verantwortlichen in Krankenversicherung und Ärzteschaft sollte es folglich darauf ankommen, gerade dieser Personengruppe die neuen Behandlungsprogramme besonders eindringlich nahe zu bringen und sie zur Teilnahme daran zu bewegen. Dazu aber bedarf es, wie die Analysen zeigen (Tab. 3), mehr als nur der Information über das Vorhandensein solcher Programme. Vermutlich waren die Krankenkassen gut beraten, als sie beschlossen, die DMP-Teilnahme ihrer Versicherten mit Erlassen der Praxisgebühr zu honorieren.

\section{Danksagung}

Die Untersuchung wurde gefördert durch die GSF.

Der Artikel nimmt besonderen Bezug auf folgende Beiträge dieses Sonderheftes von Das Gesundheitswesen: [25-35]. 
${ }^{19}$ Berger J, Slezak J, Stine N et al. Economic impact of a diabetes disease management program in a self-insured health plan: early results. Disease Manag 2001; 2: 65-73

${ }^{1}$ Bruckenberger E. Vernetzte Versorgungsmodelle mit Kliniken, Praxen und Kassen. Z Allg Med 1998; 74: 259-262

2 Janßen HJ. Kooperation und Vernetzung im Gesundheitssystem. In: Dahme HJ, Wohlfahrt N (Hrsg). Netzwerkökonomie im Wohlfahrtsstaat. Berlin: edition sigma, 2000: $201-215$

${ }^{3}$ Mühlbacher A. Integrierte Versorgung. Management und Organisation. Bern: Hans Huber, 2002

${ }^{4}$ Tophoven C. Der lange Weg zur integrierten Versorgung. Arb SozPol 2002; 9-10: $12-17$

${ }^{5}$ Sachverständigenrat für die Konzertierte Aktion im Gesundheitswesen. Bedarfsgerechtigkeit und Wirtschaftlichkeit, Band III: Über-, Unter- und Fehlversorgung. Gutachten 2000/2001.

${ }^{6}$ Jacobs K, Reschke P, Cassel D et al. Zur Wirkung des Risikostrukturausgleichs in der gesetzlichen Krankenversicherung. Schriftenreihe des Bundesministeriums für Gesundheit, Band 140. Baden-Baden: Nomos Verlagsgesellschaft, 2002

${ }^{7}$ Lauterbach KW, Stock S. Reform des Risikostrukturausgleichs. Disease-Management wird aktiviert. Dt Ärztebl 2001; 30: A- 1935

${ }^{8}$ Abholz H, Berger M. Ziele, Aufgaben und Nutzen - am Beispiel des „DMP Diabetes“ der KBV. Die BKK 2002; 3: 90 - 96

9 Rede des BÄK-Präsidenten Hoppe auf dem 105. Deutschen Ärztetag in Rostock am 28.5.2002.

${ }^{10}$ Löwel H, Stieber J, Koenig W et al. Das Diabetesbedingte Herzinfarktrisiko in einer süddeutschen Bevölkerung. Ergebnisse der MONICA Augsburg-Studien 1985 - 1994. Diab Stoffwech 1999; 8: 11 - 21

${ }^{11}$ Janka HU, Redaelli M, Gandjour A et al. Epidemiologie und Verlauf des Diabetes mellitus in Deutschland. In: Scherbaum WA, Lauterbach KW, Renner R (Hrsg). Evidenzbasierte Diabetes-Leitlinien DDG Deutsche Diabetes-Gesellschaft, 2000

${ }^{12}$ Gandjour A, Lauterbach K. European comparison of costs and quality in the prevention of secondary complications in type 2 diabetes mellitus (2000 - 2001). Diabetic Medicine 2002; 7: 594-601

13 Jönsson B. Revealing the cost of type 2 Diabetes in Europe. Diabetologia $2002 ; 7: 5-12$

${ }^{14}$ Clarke P, Gray A, Legood R et al. The impact of diabetes-related complications on healthcare costs: results from the United Kingdom Prospective Diabetes Study (UKPDS). Diabetic Medicine 2003; 6: $442-450$

15 Prof. E. Standl, Diabetologe und Chefarzt am Krankenhaus MünchenSchwabing, am 26.5.02. Nordlicht aktuell (Mitteilungsblatt der KVSH) 2002; 6: 14

${ }^{16}$ Raspe H, Sawicki P, Schmacke N. Sind vorliegende Disease-Management-Programme für Diabetes wirklich unnötig? G \& G Wissenschaft 2004; 4: $23-31$

${ }^{17}$ Häussler B, Berger U. Blühende Landschaften. Die Effektivität von Disease-Management-Programmen in der Wahrnehmung deutscher EBM-Experten. Gesundh SozPol 2004; 5-6: 48-55

18 Blonde L. Disease management approaches to type 2 diabetes. Manag Care 2000; 8: $18-23$

${ }^{20}$ Gilmer T, O"Connor PJ. Cost effectiveness of diabetes mellitus management programs: a health plan perspective. Disease Manag Health Out 2003; 7: 439-453

${ }^{21}$ Erler A. Die Einführung von Disease-Management-Programmen in Deutschland im Spiegel unterschiedlicher Interessenlagen von Krankenkassen und Kassenärztlichen Vereinigungen. Gesundheitswesen 2002; 64 (11): $572-577$

${ }^{22}$ Gerlach FM, Beyer M, Szecsenyi J et al. Evaluation von Disease-Management-Programmen. Aktuelle Defizite, Anforderungen, Methoden. Z Ärztl Fortb Qualität 2003; 97 (7): 495 - 501

23 Schulze J. Disease-Management-Programm Diabetes mellitus Typ 2. Heftige Kritik der teilnehmenden Ärzte. Dt Ärztebl 2004; 9: 543 -544

${ }^{24}$ Schmidt D. DMP...der Anfang ist gemacht? Gesundh Sozpol 2003; 5-6: $39-42$

${ }^{25}$ Holle R, Happich M, Wichmann HE. A research platform for population based health research. Gesundheitswesen 2005; 67 S1: S19-S25

${ }^{26}$ Löwel H, Döring A, Schneider A et al. The MONICA Augsburg surveys basis for prospective cohort studies. Gesundheitswesen 2005; $67 \mathrm{S1}$ : S13-S18

${ }^{27}$ Wichmann HE, Gieger C, Illig T. KORA-gen - Resource for population genetics, controls and a broad spectrum of disease phenotypes. Gesundheitswesen 2005; 67 S1: S26-S30

${ }^{28}$ Baumert J, Ladwig KH, Döring A et al. Zeitliche Veränderungen und Einflussfaktoren des Rauchverhaltens im Hinblick auf die Umsetzung von Präventionsmaßnahmen. Gesundheitswesen 2005; 67 S1: S46-S50

${ }^{29}$ Thorand B, Schneider A, Baumert J et al. Fall-Kohortenstudien. Ein effektives Design zur Untersuchung von Biomarkern als Risikofaktoren für chronische Krankheiten. Darstellung am Beispiel der MONICA/KORA Augsburg-Fall-Kohortenstudie 1984-2002. Gesundheitswesen 2005; 67 S1: S98-S102

${ }^{30}$ Meisinger C, Döring A, Heier M et al. Type 2 Diabetes mellitus in Augsburg - an epidemiological overview. Gesundheitswesen 2005; 67 S1: S103-S109

${ }^{31}$ Rathmann W, Haastert B, Icks A et al. The diabetes epidemic in the elderly population in western Europe: data from population-based studies. Gesundheitswesen 2005; 67 S1: S110-S114

32 Herder C, Illig T, Rathmann W et al. Inflammation and Type 2 Diabetes: results from KORA Augsburg. Gesundheitswesen 2005; 67 S1: S115-S121

${ }^{33}$ Illig T, Bongardt F, Schöpfer-Wendels A et al. Genetics of type 2 diabetes: impact of Interleukin-6 gene variants. Gesundheitswesen 2005; 67 S1: S122 -S126

${ }^{34}$ Mielck A, Reisig V, Rathmann W. Health inequalities among persons with type 2 diabetes: The example of intermittent claudication. Gesundheitswesen 2005; 67 S1: S137-S143

${ }^{35}$ Icks A, Rathmann W, Haastert B et al. Cost-effectiveness of type 2 diabetes screening: results from recently published studies. Gesundheitswesen 2005; 67 S1: S167-S171 